\title{
Day Case Rhinoplasty
}

\author{
GK Banfield, D McKiernan, AE Hinton
}

\begin{abstract}
Rhinoplasty is considered by many to be an inpatient surgical procedure. This may be because the operation is thought to be traumatic with a risk of epistaxis and periorbital haematoma. Since 1992 rhinoplasty surgery at St George's hospital has been routinely performed on a planned day case basis. The hospital records of 97 patients were examined of which 17 patients $(18 \%)$ underwent planned admission and 12 (12\%) unplanned admission. No patients were re-admitted to hospital after discharge. With the advances in day case surgical practice within the UK we consider that the practice of day case rhinoplasty is likely to have its place. Such surgery should ideally be performed in a dedicated day case unit and provision for admission overnight should be available.
\end{abstract}

\section{Key Words}

Day Case, Rhinoplasty

\section{Introduction}

An increasing number of surgical procedures are being performed on a day case basis. Day case surgery is seen as a method of improving efficiency and reducing expenditure. The policy has advantages for patients allowing them an early return to their familiar environment.

Many ENT procedures including tonsillectomy (1), septoplasty (2) and myringoplasty are now routinely performed in some centres on a planned nonresidential basis. However rhinoplasty is still considered by many to be an inpatient surgical procedure and there has been little to challenge this view in the literature.

At St George's hospital rhinoplasty surgery has for several years been performed on a planned day care basis. The aim of this study was to determine the outcome of this policy and assess the suitability of rhinoplasty as a day case procedure.

\section{McKiernan FRCS}

AE Hinton FRCS
Department of ENT
St George's Hospital
Blackshaw Road
London
SW17 0QT

\section{Methods}

Patients are selected for day case rhinoplasty according to established medical and social criteria (Table 1) and assessed in a pre-operative clinic. Those patients who fail to meet the day case criteria undergo surgery on a planned inpatient basis. Patients who fulfil the selection criteria are admitted on the day of operation and preferably undergo surgery on a morning list.

Some of the operations were performed in a dedicated day case unit, however, the majority of cases were admitted onto a day case annex of the main ENT ward. Routine septal quilting is performed in all cases at completion of surgery and, in the absence of bleeding, packs are removed at 2 hours post-op. At 6 hours post-op patients are assessed for haemostasis, recovery from anaesthesia and pain control. Patients are prescribed analgesia and discharged home with advice concerning possible post operative complications.

The hospital database was examined and a list of all patients who had undergone rhinoplasty between November 1992 and January 1999 was obtained. The patient notes were retrieved and unplanned overnight stay or re-admission were noted. The surgical technique together with the grade of surgeon was noted.

\section{Results}

A total of 135 patients were recorded on the hospital information systems as having undergone rhinoplasty surgery at $\mathrm{St}$ George's hospital and of these the hospital records of 97 patients were successfully located $(72 \%) .87$ patients underwent primary septorhinoplasty and 10 revision surgery. 58 patients were male and 39 female. The mean age was 34 (Range 19 $64)$.

Surgical Technique and Operating Surgeon Surgery was performed by a trainee under supervision in 61 cases $(63 \%)$ and by a consultant in 36 cases (37\%). Examination of the surgical techniques used demonstrated that osteotomies were performed in 71 cases $(73 \%)$ and tip work in 16 cases $(17 \%)$. All procedures were performed using a closed technique. Most operations, 93 (96\%) were performed on a morning list.

\section{Admission Rates}

A total of 29 patients (30\%) were kept in for overnight stay. Of these, 17 patients (18\%) underwent planned admission and 12 $(12 \%)$ unplanned admissions. Of the 17 planned admissions 14 were not considered 
medically fit for day case surgery and 3 did not meet the social criteria. Two patients had no escort and 1 was considered to live too far from the hospital.

The 12 unplanned admissions were due to continued bleeding in 6 cases. One patient bled excessively at the time of operation, another patient was recorded as losing $500 \mathrm{mls}$ of blood during the operation and was kept in hospital overnight for observation; finally the remaining 4 patients that were admitted on an unplanned basis due to bleeding were noted at post-op ward review to have a persistent ooze and the packs were retained overnight. The remaining 6 unplanned admissions were due to nausea in 3 cases, pain in 1 case and 2 patients were considered insufficiently recovered from the anaesthetic for same day discharge. No patients were re-admitted to hospital after discharge.

\section{Discussion}

Day case surgery has become steadily more popular as the cost benefits have been recognised and as the short stay has come to be accepted by the public. As a result, more efficient use is made of limited resources and more patients can be treated. Many ENT procedures are well suited to day case surgery. Many operations can be completed within an hour and do not result in debilitating pain or handicap of movement. Post-operative bed rest is short and a return to most day to day physical activities can be anticipated at an early interval.

The Royal College of Surgeons (Eng) comparative audit service (3) established the ENT day surgery rate to be $31 \%$ as reported by 80 consultants in 63 units within England and Wales. A wide range of ENT operations are reported to be performed on a day case basis including micro laryngeal surgery (4) functional endoscopic sinus surgery, ossiculoplasty and pinnaplasty. There is extensive literature supporting day case tonsillectomy $(5,6,7,8)$ and septoplasty $(9,10)$. Rhinoplasty is still considered by many an inpatient procedure probably because it is perceived to be a traumatic operation that can result in both post-operative periorbital swelling and haemorrhage. Since 1992 it has been the practice at St. George's hospital to perform rhinoplasty on a planned day case basis where possible.

\section{Admission Rates}

Twenty-nine patients (30\%) were admitted. Of these 17 patients (18\%) were admitted on a planned basis having failed to meet the day case admission criteria. There was a noticeable fall in the planned admission rate in the more recent years as day case surgery has become more accepted.

Twelve patients (12\%) were admitted on an unplanned basis and it is this group that will cause the greater concern to surgeons who might consider offering day case rhinoplasty. Out of the total series of 97 patients only 4 cases were performed on an afternoon list and 2 of these (50\%) required admission overnight. These 2 cases were not discharged home the same day as they were not considered sufficiently recovered from the anaesthetic; both patients were discharged the next morning. Two of the 12 admitted patients might, therefore, have gone home the same day if all day case rhinoplasty surgery had been performed on a morning list. Four patients were admitted overnight owing to nausea or pain. These patients were all early in the series and it could be argued that with the evolution of day case surgery within the UK, particularly anaesthetic and drug advancements (eg Ondansetron) at least some of these admissions would now be avoided. The 6 remaining cases were admitted overnight owing to haemorrhage. Two cases bled significantly during surgery and 4 were noted to have persistent "ooze" at post-op ward review necessitating retention of packs overnight. No patients were returned to theatre.

There was no noticeable pattern to these overnight stays for post-op haemorrhage and it seems unlikely that this $6 \%$ admission rate secondary to bleeding can be completely eliminated at the present time. However it should be noted that all patients requiring admission for post-operative haemorrhage were identified during the routine period of post surgery monitoring. No patient once discharged after 6 hours post-op observation required re-admission.

The Royal College of Surgeons of England Guidelines on Day Case Surgery recommends an admission rate of not more than two to three per cent (11). A $6 \%$ postoperative haemorrhage rate falls outside these recommendations and this will discourage some surgeons from undertaking day case rhinoplasty. However it should be noted that other more accepted day case procedures may fall outside these recommendations. According to the Comparative Audit Service (3) the admission rates for tonsillectomy is $14 \%$, septoplasty $8 \%$ and adenoidectomy $4 \%$.

It seems inevitable that the trend towards more and more day case surgery will continue as targeted in 1993 by The Chief Medical Officer when he set a day case surgery rate of $50 \%$ by the year 2000 (Department of Health, H93/977). To achieve this ambition further operations will need to be assessed for their suitability as non-residential basis procedures as well as accepted day case procedures being performed in greater numbers.

With the advances in day case surgical practice within the UK we consider that the 
practice of day case rhinoplasty is likely to have its place. Such surgery should ideally be performed in a dedicated day case unit and provision for admission overnight should be available. Day case rhinoplasty should be performed on a morning list and attention to surgical technique including septal quilting and short duration nasal packing is considered important.

Table 1

\begin{tabular}{l}
\hline Criteria for listing as a day case \\
\hline Medical \\
Age $<65$ years \\
ASA 1 or 2 \\
No previous general anaesthetic problems \\
Not obese \\
Not hypertensive \\
No diabetes mellitus \\
No sickle cell disease \\
Social \\
Access to a private car \\
Live less than one hour away \\
Available adult to provide care for 48 hours \\
Telephone
\end{tabular}

\section{References}

1. Hellier WPL, Knight J, Hern J, et al. Day case paediatric tonsillectomy: a review of three years experience in a dedicated day case unit Clin. Otolaryngology (1999) 24, 208 - 212

2. Hogg RP, Prior M J, Johnson AP. Admission rates, early re-admission rates and patient acceptability of 142 cases of day case septoplasty Clin. Otolaryngology (1999) 24, 213 - 215

3. Brown PM, Fowler S, Ryan R et al. Audit Article ENT day surgery in England and Wales - an audit by the Royal College of Surgeons (Eng) Comparative Audit Service The fournal of Laryngology and Otology (1998) 112, 161 - 165

4. Ah-See KW, Kelly G, Todd JG et al. A prospective evaluation of the feasibility of day case microlaryngeal surgery The fournal of Laryngology and Otology (1998) 112, 351 - 354

5. Tewary AK, Curry AR. Same-day tonsillectomy The fournal of Laryngology and Otology (1993) 107, $706-708$

6. Yardley M Editorial: Is it appropriate to perform adenoidectomy tonsillectomy or adenotonsillectomy on a day case basis? Clin. Otolaryngology (1995) 20, $95-96$

7. Chaing TM, Sukis AE, Ross DE. Tonsillectomy performed on an outpatient basis. Report of a series of 40,000 cases performed without a death Arch. Otorhinolaryngology (1968) 13, 157 - 163

8. Fenton JE, O'Dwyer TP. Adult day case tonsillectomy: a safe and viable option Clin. Otolaryngology (1994) 19, 470 - 472

9. Buckley JG, Mitchel DB, Hickey SA et al. Submucous resection of the nasal septum as an outpatient procedure The Fournal of Laryngology and Otology (1991) 105, 544 - 546

10. Nieminen P, Silvola J, Aust R et al. Nasal septal surgery as an out-patient procedure The fournal of laryngology and Otology (1997) 111, 1034 - 1037

11. Royal College of Surgeons of England (1992) Guidelines on Day Case Surgery. Revised Edition 